\title{
Radion Cosmology in Theories with Universal Extra Dimensions
}

\author{
Anupam Mazumdar ${ }^{1}$, R. N. Mohapatra $^{2}$, and A. Pérez-Lorenzana ${ }^{3}$ \\ 1 CHEP, McGill University, 3600 University Road, \\ Montréal, Québec, H3A 2T8, Canada \\ 2 Department of Physics, University of Maryland, College Park, MD 20742, USA \\ ${ }^{3}$ Departamento de Física, Centro de Investigación y de Estudios Avanzados del I.P.N. \\ Apdo. Post. 14-740, 07000, México, D.F., México
}

(Dated: October, 2001)

\begin{abstract}
We discuss cosmology of models with universal extra dimensions, where the Standard Model degrees of freedom live in a $4+n$ dimensional brane, with $n$ compact and small extra spatial dimensions. In these models, the simplest way to obtain the conventional 4-dimensional Planck scale starting with a low string scale is to have also some larger extra dimensions, where only gravity propagates. In such theories, dimensional reduction generically leads to at least two radion fields, one associated with the total volume of the extra spatial dimensions, and the other with the ratio of the sizes of small and large extra dimensions. In this paper, we discuss the impact of the radion fields on cosmology. We emphasize various aspects of radion physics such as radion coupling to the Standard Model fields, bare and dressed radion masses during inflation, dynamical stabilization of radions during and after inflation, radion decay life time and its late dominance in thermal history of the Universe as well as its quantum fluctuations during inflation. We argue that models where the radion plays the role of an inflaton or the inflaton is a brane scalar field, run into problems. We then present a successful inflation model with bulk scalar fields that seems to have all the desired properties. We also briefly discuss the possibility of radion as a cold dark matter candidate.
\end{abstract}




\section{INTRODUCTION}

The idea that the universe may have extra spatial dimensions has been under consideration for many years. The earliest thoughts date back to the works of Kaluza and Klein in the 1920's. Such a possibility has been lately reinforced by the advent of string theory which is believed to be the most attractive candidate for a theory of gravity and gauge interactions [1], where the simplest realization requires at least 10 dimensions to be consistent. The extra dimensions are usually assumed to be compact with the sizes of the order of the fundamental Planck scale, $l_{p}=M_{p}^{-1}=\left(2.436 \times 10^{18}(\mathrm{GeV})^{-1} \sim 10^{-33} \mathrm{~cm}\right.$. However recent developments 2] in this field have shown the prospects of having large compact dimensions [3]. The largest possible compact dimension could be as large as a few micrometers [4]. In the simplest incarnation it is believed that the Standard Model (SM) fields live in a four dimensional hypersurface or brane embedded in a higher dimensional space, which is known as the bulk, while gravity can propagate in all dimensions (for some earlier ideas see also Refs. [5]).

In these models gravitational interactions appear to be stronger when probing distances below the size of the extra dimensions. The actual fundamental scale of gravity, $M_{*}$, is no longer the (now effective) 4 dimensional Planck scale, but it is rather related to this by [4]

$$
M_{p}=M_{*}^{2+d} \cdot \operatorname{vol}
$$

where vol denotes the volume of the $d$ compact spatial dimensions. Current measurements on the precision of the Newton's law at small distances impose an upper bound on the size of the extra dimensions at a level of 100 microns $[6]$. For $d=2$ it has been pointed out that the fundamental scale of quantum gravity could be as low as few $\mathcal{O}(\mathrm{TeV})$, which will have interesting implications for collider physics [7, 8, 9]. From this perspective these theories have an edge of being testable in future terrestrial and extra terrestrial experiments. The models with extra dimensions also provide an unique geometric insight to the naturalness problem in physics, which is one of the greatest reasons for studying various aspects of these theories in particle physics and in cosmology and astrophysics. Actually the stringent bounds on the size of the extra dimensions, or equivalently on the fundamental scale have been obtained from the astrophysical implications of the excited states of graviton, $M_{*}>1000 \mathrm{TeV}[10]$. However very recent study reveals that such a bound can be weakened down to few TeV when the momenta associated with the extra dimensions are broken [11]. 
Another class of extra dimension models proposes that the SM degrees of freedom also propagate in some of the extra dimensions; examples of such studies can be found in Refs. 12, 13, 14, 15, 16, 17, 18, 19, 20]. In this scenario, each SM particle is accompanied by a Kaluza Klein $(\mathrm{KK})$ tower, with masses spaced by the inverse size of the extra dimensions in which they reside. Since there is no evidence of a KK tower associated with any SM particle yet, the size of such extra dimensions has to be smaller compared to the inverse of the electroweak scale, $\sim \mathrm{TeV}^{-1}$. These models go by the name of universal extra dimension models (UED). Amongst the interesting features of these models is the possibility that they can be tested in near future in collider experiments through direct production of the excited KK states of the standard model particles [14]. Furthermore in six dimensions, such scenarios acquire the additional virtue that they can provide a natural explanation for the number of generations [15, 16], and an understanding of proton stability [17, 18] along with the lightness of the observed neutrino masses [18, 19], despite the absence of any large mass scales in the theory. These models are therefore phenomenologically more sound than models where the standard model resides in $3+1$ dimensional Minkowski brane.

Cosmology of models with large extra dimensions, where only gravity propagates in the bulk has received a great deal of attention, from building inflationary models 21, 22, 23, 24, 25, 26] to studying moduli problem [27], moduli stabilization [23], thermal history [28], and baryogenesis [29]. In this paper our main aim is to address similar cosmological issues in the class of models where both standard model particles and gravity propagate in the bulk (or the UED models).

For simplicity we consider the $n$ extra spatial dimensions where the standard model particles propagate, compactified on orbifold-ed circles with a common size $r$. The power law running of the gauge couplings in these models [12, 13] and the need for keeping the theory from violating unitarity bounds [14], suggest that $r$ should not be much larger than about 10-100 times the inverse fundamental scale $\left(M_{*}^{-1}\right)$, i.e. $M_{*} r \sim \mathcal{O}(10)-\mathcal{O}(100)$. Explaining the weakness of gravity in such theories (where $M_{*}$ of order of $10 \mathrm{TeV}$ ), requires that there must be large extra dimensions orthogonal to the $4+\mathrm{n}$ already considered [4] (i.e. orthogonal to the SM brane).

In the simplest scenario, we consider UED models comprising two sets of asymmetric extra spatial dimensions: $n$-small dimensions, of a size $r$ just around a factor of ten to hundred times above the fundamental scale $M_{*}^{-1}$, and $p$-larger spatial dimensions of size $R$. 
Note that the SM fields are glued to $4+n$ dimensions only, while gravity is propagating in the entire bulk. Therefore the effective four dimensional Planck mass $M_{p}$ is given by

$$
M_{p}^{2} \equiv M_{*}^{2+n+p} r^{n} R^{p}
$$

For $M_{*}$ as low as few $\mathrm{TeV}$, there should be at least two large extra spatial dimensions of sub millimeter size in order to yield the correct four dimensional Planck scale.

In the above mentioned set-up, upon dimensional reduction, there are at least two radions. One describes the variations of the whole volume of the extra dimensions. We will call it the volume radion. The other, here called the shape mode, describes the relative expansion and contraction of the two sets of extra dimensions. In this paper we will take a phenomenological approach in determining the masses of the two radions, which could be either light or heavy compared to cosmological scale, which is usually taken to be the Hubble parameter. We will show that these radions play an extremely important role in determining the fate of the early Universe in UED models.

The early universe cosmology is incomplete if it cannot reproduce the successes of the hot big bang model, e.g. synthesis of light elements at a scale $\sim 1 \mathrm{MeV}$ and their present day observed abundance. However this cannot be reached if the universe prior to this era were not already filled by the SM degrees of freedom, and provided there were no excess generation of entropy after big bang nucleosynthesis (BBN), which would otherwise dilute the initial abundance. This poses a challenge to any particle physics theory with large extra dimensions. In this regard UED models are no exception. They come with light scalar fields and therefore it is paramount that we study the dynamics and decay life time of these radions. The late oscillations and eventual decay of the radions can dominate thermal history of the universe, which can pose a serious threat to BBN. This is analogous to the string moduli problem. Therefore it is important that radions either decay before BBN, via their couplings to the SM fields, or they are sufficiently light to survive the age of the universe. If they survive late then they can be regarded as candidates for cold dark matter. In an ideal situation it would be possible to stabilize both the volume and the shape modes during inflation and/or right after the end of inflation. This is a major issue which we will deal in some detail in our paper. We argue that only the volume radion can be stabilized dynamically during inflation, because it always couple to the inflaton, whereas the shape mode remains uncoupled to it. Besides this, there is another important difference: the 
volume radion governs the four dimensional Newton's constant, therefore it is important that we stabilize this radion before BBN. There are already stringent bounds on variations on the Newton's constant during BBN [30]. On the other hand the shape mode can remain light and dynamically active field. We will find that it must also be stabilized before BBN. The shape mode can give rise to the running of the SM coupling constants.

Furthermore, we also describe a successful inflationary scenario in UED model. We will discuss three possibilities, radion driven inflation, brane field driven inflation and the bulk field driven inflation and assess the relative merits of the different scenarios.

Light scalars in cosmology are sometimes regarded as boon if they can sustain a flat potential with a large vacuum energy since they can then give rise to accelerated expanding phase of the universe, the inflation phase. They can also dilute all energy densities except the quantum fluctuations which can be imprinted on the cosmic microwave background radiation. The radions in UED models could be responsible for generating adiabatic density fluctuations seen by COBE [31], BOOMERANG 32], MAXIMA [33], DASI 34] and WMAP [35, 36]. In this paper we propose that even if the radions are not the ideal candidate for inflaton, they can still be responsible for reheating the universe with the SM degrees of freedom and also converting its fluctuations into adiabatic modes.

This paper is organized as follows. We begin by introducing our general set-up and performing a dimensional reduction of the gravitational part of the action in order to identify the radion fields. In the subsequent sections we devote our discussion on general aspects of the radion physics; we introduce the radion to matter couplings in section 3; we discuss initial conditions of the radion vacua before the onset of inflation in section 4 . In section 5, we comment on the problems of dealing with the radion (stabilization) potential and the radion mass, which seem to have an important effect on the dynamics of inflation and thermal history of the universe. Then we discuss the decay rate and life time of the radion fields. We begin section 6 with the analysis of the potential role of the radions in the early Universe. First we discuss the case when the radion acts as an inflaton. Then we extend our scenario by including a brane or a bulk inflaton. Then we discuss a possible role of radions to generate SM relativistic degrees of freedom along with the adiabatic density perturbations. We conclude our discussion by mentioning the possibility for the radions to become a candidate for the cold dark matter. 


\section{THE SET-UP, DIMENSIONAL REDUCTION AND THE TWO RADIONS}

Let us consider the following metric in $4+d$ dimensions

$$
d s^{2}=G_{A B} d x^{A} d x^{B}=g_{\mu \nu} d x^{\mu} d x^{\nu}-h_{i j} d y^{i} d y^{j}
$$

with $A, B$ running over $\mu$ and $i$, where $\mu=0,1,2,3$ labels our usual four dimensions and $i, j=1, \ldots, d$ are the total $d$ extra dimensions. The coordinates $y^{i}$ take values in the interval $[0,1]$. Note that since we have taken $y^{i}$ to be dimensionless, our $h_{i j}$ has length dimension two and $h^{i j}$ has $L^{-2}$.

Also note that we are not considering the presence of vector-like connection $A_{\mu}^{i}$ pieces, which are common in Kaluza Klein theories. This is because we are only interested in the zero mode part of the metric, after orbifolding the extra spatial dimensions. We have $A_{\mu}^{i}$ odd under parity transformation and therefore it vanishes at the zero mode level. By reducing the $4+d$ scalar curvature term, we obtain at zero mode level the effective action

$$
\begin{aligned}
S & =\frac{1}{\kappa_{*}^{2}} \int d^{4} x d^{d} y \sqrt{-G} R[G] \\
& =\frac{1}{\kappa^{2}} \int d^{4} x \sqrt{-g} \frac{\sqrt{h}}{v_{0}}\left\{R[g]-\frac{1}{4} \partial_{\mu} h^{i j} \partial^{\mu} h_{i j}-\frac{1}{4} h^{i j} \partial_{\mu} h_{i j} \cdot h^{k l} \partial^{\mu} h_{k l}\right\}
\end{aligned}
$$

where $1 / \kappa_{*}^{2}=M_{*}^{2+d} / 2$ defines the true fundamental gravity scale, while $1 / \kappa^{2}=M_{p}^{2} / 2$, defines the effective 4 dimensional Planck scale, which is related to the fundamental scale by a volume suppression, $\kappa_{*}^{2}=\kappa^{2} \cdot v_{0}$, see Eq. (1.2). Here $v l_{0}$ is the stabilized volume of the extra space and corresponds to stabilized values for the $h_{i j}$.

In order to obtain the 4 dimensional scalar curvature term in a canonical form, we have to perform a conformal transformation on the metric,

$$
g_{\mu \nu} \rightarrow e^{2 \varphi} g_{\mu \nu}
$$

designed to cancel the extra $\sqrt{h} /$ vol $_{0}$ coefficient of $R[g]$ in Eq. (2.2). We take $\varphi$ such that

$$
e^{2 \varphi} \sqrt{h} / \operatorname{vol}_{0}=1
$$

The action in Eq. (2.2) is then transformed into

$$
S=\frac{1}{\kappa^{2}} \int d^{4} x \sqrt{-g}\left\{R[g]-\frac{1}{4} \partial_{\mu} h^{i j} \partial^{\mu} h_{i j}+\frac{1}{8} h^{i j} \partial_{\mu} h_{i j} \cdot h^{k l} \partial^{\mu} h_{k l}\right\} .
$$


Next for the four dimensional part of the metric, $g_{\mu \nu}$, we will assume the standard FriedmanRobertson-Walker metric with a flat geometry i.e.

$$
g_{\mu \nu}=\operatorname{diag}(1,-a(t),-a(t),-a(t))
$$

for an isotropic and homogeneous (brane) universe, whereas we consider a diagonal form for the $h$ part of the metric:

$$
h_{i j} \leftrightarrow \operatorname{diag}\{\overbrace{h_{1}^{2}, \ldots, h_{1}^{2}}^{n \text {-times }}, \overbrace{h_{2}^{2}, \ldots, h_{2}^{2}}^{p \text {-times }}\}
$$

where we have assumed $n(p)$ of the $d$ extra dimensions to be small (large) with common physical sizes, $h_{1}(t)$ and $h_{2}(t)$ respectively. Obviously $d=n+p$, and the physical volume of the extra space is given as

$$
v o l_{\text {phys }}=\sqrt{h}=h_{1}^{n} h_{2}^{p} .
$$

At present, the physical sizes of the extra dimensions are given by $h_{1,0}=r$ and $h_{2,0}=R$, where the zero subscript stands for the stabilized values. Therefore the stabilized volume is given by $v_{0} l_{0}=r^{n} R^{p}$, as stated in Eq. (1.2).

With the help of Eqs. (2.7) and (2.5) we obtain the non diagonal kinetic terms to be

$$
\frac{1}{2 \kappa^{2}}\left\{n(n+2)\left(\frac{\partial_{\mu} h_{1}}{h_{1}}\right)^{2}+2 n p\left(\frac{\partial_{\mu} h_{1}}{h_{1}}\right)\left(\frac{\partial^{\mu} h_{2}}{h_{2}}\right)+p(p+2)\left(\frac{\partial_{\mu} h_{2}}{h_{2}}\right)^{2}\right\} \text {. }
$$

We diagonalize this by introducing two physical radion fields

$$
\sigma_{v}=M_{p} \sqrt{\frac{n+p+2}{2(n+p)}} \ln \left(\frac{\sqrt{h}}{v_{o l}}\right), \quad \sigma_{\perp}=M_{p} \sqrt{\frac{n p}{n+p}} \ln \left(\frac{h_{2}}{h_{1}} \frac{r}{R}\right) .
$$

This makes the kinetic terms in Eq. (2.8) diagonal and canonically normalized. Both the radions have a straightforward physical interpretation, $\sigma_{v}$ is a sole function of $\sqrt{h}$, therefore it is related to the variation of the physical size of the volume, and often called a volume modulus (or volume radion). On the other hand $\sigma_{\perp}$ is related to the ratio of the sizes of both large and small dimensions, and we will often call it the shape mode. Furthermore, note that both the radion fields are set to be zero when stabilized (that is when $h_{1}$ and $h_{2}$ obtain the values $r$ and $R$ respectively).

The effective four dimensional action for the gravity sector can then be written as

$$
S=\int d^{4} x \sqrt{-g}\left\{\frac{1}{\kappa^{2}} R[g]+\frac{1}{2}\left(\partial_{\mu} \sigma_{v}\right)^{2}+\frac{1}{2}\left(\partial_{\mu} \sigma_{\perp}\right)^{2}-U\left(\sigma_{v}, \sigma_{\perp}\right)\right\} .
$$


Note that in a particular case when $n=0, \sigma_{\perp}=0$ and we are reduced to a single radion scenario of large extra dimensions. The last term in the above action represents the radion potential that must be introduced by hand to stabilize the extra dimensions. Its origin is largely unknown so far. In this paper we will only assume that there exists such a potential $U\left(\sigma_{v}, \sigma_{\perp}\right)$ and consider illustrative forms for it in our discussion. As we will discuss later on, the detailed form of $U\left(\sigma_{v}, \sigma_{\perp}\right)$ is important in understanding the dynamics of the radions during and after inflation.

\section{RADION COUPLINGS}

Given the above definitions for the radions, we now discuss how they couple to matter, both in the bulk and on the brane. We start by considering a bulk scalar field, $\tilde{\phi}$ which latter on could be identified as the inflaton. The action, before performing the conformal transformation on the metric, goes as

$$
S_{\tilde{\phi}}=\int d^{4} x d^{n+p} y \sqrt{-g} \sqrt{h}\left[\frac{1}{2} G^{A B} \partial_{A} \tilde{\phi} \partial_{B} \tilde{\phi}-V_{b u l k}(\tilde{\phi})\right]
$$

We now take only the zero mode level of the theory, therefore we write $\tilde{\phi}(x, y)=\phi(x) / \sqrt{v_{0} l_{0}}$, and properly scale the potential by introducing an effective four dimensional potential, $V(\phi)=\operatorname{vol}_{0} \cdot V_{\text {bulk }}(\tilde{\phi})$. By integrating out the extra dimensions and introducing the conformal transformation, we obtain an effective scalar action in the Einstein frame,

$$
S_{\phi}=\int d^{4} x \sqrt{-g} e^{2 \varphi} \frac{\sqrt{h}}{v o l_{0}}\left[\frac{1}{2} g^{\mu \nu} \partial_{\mu} \phi \partial_{\nu} \phi-e^{2 \varphi} V(\phi)\right] .
$$

It is now straightforward to see that the last term gives rise to a coupling of $\phi$ to $\sigma_{v}$ due to the definition of $\varphi$ given in Eq. (2.4). This will also take care of the global exponential term, which cancels the $\sqrt{h} / v_{0} l_{0}$ piece of the action. Therefore we can simply write

$$
S_{\phi}=\int d^{4} x \sqrt{-g}\left[\frac{1}{2} g^{\mu \nu} \partial_{\mu} \phi \partial_{\nu} \phi-e^{-\alpha \sigma_{v} / M_{p}} V(\phi)\right]
$$

where the coupling constant is given by

$$
\alpha=\sqrt{\frac{2(n+p)}{n+p+2}} .
$$

It is worth stressing that the $\sigma_{\perp}$ radion does not couple to the bulk scalar field. Furthermore, since the radion couplings appear due to the conformal transformation in Eq. (2.3), 
which depends only on $\sigma_{v}$, we can conclude the same is actually true for any bulk field. Therefore the $\sigma_{\perp}$ radion will only couple to the brane matter, which we shall discuss now. Note that at this level we already notice an important difference in the dynamics of the two radion fields.

Let us now consider a brane scalar field, $\tilde{\chi}$. The general action would be given as in $4+n$ dimensions, where the SM fields propagate. This means that we have already integrated out extra $p$ large dimensions which are harmless for the brane fields. Once more we stay at the zero mode level, we introduce a properly normalized zero mode by defining $\tilde{\chi}=\chi / \sqrt{r^{n}}$. We start with the effective action

$$
S_{\chi}=\int d^{4} x \sqrt{-g}\left(\frac{h_{1}}{r}\right)^{n}\left[\frac{1}{2} g^{\mu \nu} \partial_{\mu} \chi \partial_{\nu} \chi-V(\chi)\right] .
$$

Notice that unlike the bulk field case, the induced metric does not contain the whole $\sqrt{h}$, but only the piece that goes along the extra dimensions of the brane, $h_{1}^{n}$. Therefore after conformally transforming the metric there will be a remnant of the $h$ metric. Indeed by writing $h_{1}^{n}=\sqrt{h} h_{2}^{-p}$ and then, using the radion definitions in Eq. (2.9) in order to replace the $h_{2}$ term, we obtain

$$
S_{\chi}=\int d^{4} x \sqrt{-g} e^{-\beta \sigma_{v} / M_{p}} e^{-\gamma \sigma_{\perp} / M_{p}}\left[\frac{1}{2} g^{\mu \nu} \partial_{\mu} \chi \partial_{\nu} \chi-e^{-\alpha \sigma_{v} / M_{p}} V(\chi)\right] .
$$

where the coupling constants are given by

$$
\begin{aligned}
& \beta=\frac{p}{\sqrt{(n+p)(n+p+2)}}, \\
& \gamma=\sqrt{\frac{n p}{n+p}} .
\end{aligned}
$$

We now see that the $\sigma_{\perp}$ radion has a global coupling to both kinetic and potential terms of the brane fields, whereas extra global coupling of $\sigma_{v}$ to both kinetic and potential terms also appear. Such couplings affect the definition of the canonical brane field, which are, however, nicely recovered around the minima of $\sigma_{v, \perp}$.

As far as the other fields go, following a similar procedure, we find that the radion couplings to brane matter are given as:

$$
S_{\text {gauge }}=-\int d^{4} x \sqrt{-g} e^{(\alpha-\beta) \sigma_{v} / M_{p}} e^{-\gamma \sigma_{\perp} / M_{p}} \frac{1}{4} F_{\mu \nu} F_{\rho \tau} g^{\mu \rho} g^{\nu \tau},
$$

for the zero mode gauge fields, and by

$$
S_{\psi}=\int d^{4} x \sqrt{-g} e^{-(\alpha / 2+\beta) \sigma_{v} / M_{p}} e^{-\gamma \sigma_{\perp} / M_{p}}\left(-i \bar{\psi} D_{\mu} \gamma_{a} \psi\right) e_{\nu}^{a} g^{\mu \nu}
$$


for the zero mode fermions. It is worth mentioning that if we take $n=0$, as for the case of large extra dimensions, we find that $\alpha=\beta$, and thus the radion coupling to a massless gauge field vanishes [7]. This is however not the case when the matter fields propagate only in part of the extra space as shown in Eq. (3.9).

\section{INITIAL CONDITIONS BEFORE INFLATION}

The most natural scale for the universe to start in $4+d$ dimensions is the fundamental scale. Therefore, by taking $h_{1,2}(t=0) \sim M_{*}^{-1}$ as the initial values for the size of the extra dimensions, we obtain the initial vacuum expectation values (vevs) for $\sigma_{v}$ and $\sigma_{\perp}$ are of the order of the Planck scale:

$$
\sigma_{v}(0)=-2 M_{p} \sqrt{\frac{n+p+2}{n+p}} \ln \left(\frac{M_{p}}{M_{*}}\right), \quad \sigma_{\perp}(0)=-M_{p} \sqrt{\frac{n p}{n+p}} \ln \left(\frac{R}{r}\right) .
$$

Note that both the initial values for the radions are negative. This is due to the fact that the extra dimensions are relatively larger today (and so the volume). Physically, the extra dimensions have to grow, while both the radions approach to zero from negative values. For the sake of illustration we can take $M_{*} \sim \mathcal{O}(\mathrm{TeV})$, and we find that indeed both the absolute values for the vevs, though $n$ and $p$ dependent, will have initial vevs quite large, e.g. $\sigma_{v}(0) \sim \mathcal{O}\left(10 M_{p}\right), \sigma_{\perp}(0) \sim \mathcal{O}\left(10 M_{p}\right)$.

There is a couple of interesting points to be mentioned. First, the observed Universe is many orders of magnitude larger than $M_{*}^{-1}$, and it is also certainly larger than even the largest $p$ extra dimensions, which should be smaller than few micrometers in order to evade detection at small distance gravity experiments. Therefore an asymmetric expansion of the spatial dimensions is required. Besides the galaxy formation requires that there has to be perturbations seeded on large scales (larger than the size of the horizon). Inflation is the only paradigm which makes the universe large, flat, isotropic and homogeneous, if it began at $M_{*}^{-1}$. Even though it creates a homogeneous and isotropic universe, it cannot really beat the quantum fluctuations which are being stretched outside the horizon at the time of inflation. These quantum fluctuations when they reenter into our horizon late during the radiation dominated era provide the seed for the galaxy formation [37].

However what remains to be seen is what could be the inflaton in our scenario. We should also remind the reader that at the moment fundamental theory provides no guidance 
regarding the radion potential and, therefore the masses for $\sigma_{v}, \sigma_{\perp}$ are not clearly fixed by our action Eq. (2.10). This leads to a degree of speculation which we will discuss briefly.

\section{RADION MASS}

Although, from a naive point of view one would expect that the radion masses ought to be somehow related to the compactification scale, this seems to be less obvious when we recognize that physical radions in an asymmetric bulk are not solely connected to a particular extra dimension, but a combination of all. Moreover, since we have no knowledge about the origin and actual form of the stabilization potential, very little can be said about the radion masses without further assumptions. It has been conjectured that the radion mass can be as small as $m_{r} \lesssim M_{c}^{2} / M_{p}$ [38] for $M_{c}=1 / r$. This seems certainly true for the case where the stabilization potential comes from brane physics, however the mass can be much larger if bulk physics plays a role as we argue below.

For the case where the stabilization potential is based on bulk physics there is a volume enhancement in the effective four dimensional theory. On pure dimensional grounds, we can see that the potential could be as large as $M_{*}^{2} M_{p}^{2}$, rather than $M_{*}^{4}$ leading to much larger radion masses. For instance, in this case, we could write the radion potential roughly as $U(\sigma) \lesssim M_{*}^{2} M_{p}^{2} U\left(\sigma / M_{p}\right)$, so that the upper bound on the radion mass comes naturally as $m_{r}^{2} \lesssim M_{*}^{2}$. Another intuitive way of seeing this result is by noticing that masses for the radions $m_{r}^{2}{ }_{i} \sigma_{i}^{2}$, where $i=v, \perp$, are invariant under dimensional reduction. The possibility of having a large radion mass has also been pointed out in another example given in Ref. [39], where radion mass and compactification scale are exponentially related to each other, therefore reconciling the likelihood of large dimensions with large radion masses.

As it has already been noticed [38] a light mass radion can mediate long range interactions with a similar strength as gravity, therefore, the experiments testing the Newton's law at small distances [6] impose a lower bound on such radion masses, which corresponds to the mass scale, $m_{r}>10^{-3} \mathrm{eV}$. 


\section{A. A phenomenological guess on radion potential}

While we have no guidance from a fundamental theory regarding the nature of the radion potential, there are some cosmological constraints which could be used to guess its overall nature. First of all, we would like to have the minimum of the radion potential at $\sigma_{v}=0$ and $\sigma_{\perp}=0$. Secondly, since the volume modulus, $\sigma_{v}$ governs the magnitude of the Newton's constant, it must be stabilized before $\mathrm{BBN}$, in order to be compatible with stringent bounds on the variation of the Newton's constant during BBN [30]. The shape mode must also be stabilized in order to evade constraints from long range interaction between the SM fields and late time variations of coupling constants. Since the stabilization crucially depends on the actual form of the stabilization potential, this provides some constraint on its form.

The two extreme possibilities for the potentials are as follows:

(i) The potential for the radions could be fairly flat, even for larger vevs, in which case a simple mass squared-like potential, e.g. $U \sim \frac{1}{2} m_{r}^{2} \sigma_{r}^{2}$, where $r=v, \perp$ can be used as a phenomenological model. As we will show this choice of potential can be extremely hard to stabilize. Besides the late oscillations of the radions will give rise to a moduli problem, for which there is no simple solution.

(ii) This problem can be ameliorated provided that the moduli potential is steeper than $m_{i}^{2} \sigma_{i}^{2}$ at large vevs, or in other words, the flatness of the potential is lifted. Let us consider for example a phenomenological potential that can give rise to a volume stabilization

$$
U(v o l) \sim \mu^{2} M_{*}^{2+2(n+p)}\left(v_{0 l}-v_{0 l} l_{p h y s}\right)^{2},
$$

where the overall scale has been taken in the general philosophy of using $M_{*}$ to adjust the dimensions of the potential. Here $\mu$ is assumed to be some mass scale naturally

related to the volume, and so this is at most of order $M_{*}^{2} / M_{P}$. By using Eq. (1.2), we can rewrite the potential as

$$
\left.U(v o l)=m_{v}^{2} M_{p}^{2}\left(1-\operatorname{vol}_{\text {phys }} / \text { vol }_{0}\right)^{2}=m_{v}^{2} M_{p}^{2}\left(1-\sqrt{h} / v_{0}\right)_{0}\right)^{2}
$$

where now the mass scale is $m_{v}=\mu M_{P} / M_{*}$, which is much larger than $\mu$, but yet smaller than $M_{*}$. The above equation translates into a potential for the volume mod- 
ulus with an exponential profile:

$$
U\left(\sigma_{v}\right) \sim m_{v}^{2} M_{p}^{2}\left(1-e^{\alpha_{v} \sigma_{v} / M_{p}}\right)^{2}
$$

Furthermore, note that the radion mass around the minimum becomes $m_{v}$, therefore reinstating our arguments. Some interesting facts about the above potential are that (i) it is exponentially steep for $\sigma_{v}>0$, which means that such a potential will not allow the volume to grow much larger than its stable size. (ii) On the other hand, the potential is fairly flat for large and negative vevs of $\sigma_{v}$, allowing the volume to easily expand. Let us stress here that we are simply using this potential for illustrative purposes.

Needless to mention that the above arguments should also hold for the shape mode. However unlike the volume modulus it is extremely hard to make any speculation on the mass for the shape mode. The only obvious constraint comes from the long range interaction mediated by $\sigma_{\perp}$, which gives the lower bound $m_{\perp} \geq 10^{-3} \mathrm{eV}$, as already mentioned. In summary we can safely argue that the mass range for both the radions could be

$$
10^{-3} \mathrm{eV} \leq m_{r} \leq M_{*},
$$

although there are models where the mass of the volume modulus radion could easily be close to the upper bound.

\section{DECAY RATES OF THE TWO RADIONS}

Massive radions may decay into visible particles. Decay channels may vary depending on the actual mass. However, without loss of generality we can say that radions decay into photons, and possibly other SM particles. The couplings that are responsible for such

processes are described in section three. In all cases, but when the SM fields are truly four dimensional (when $n=0$ ), the decay would take place at tree level and, when evaluated around the stabilized radions they will always come with Planck suppressed couplings. The decay rate for any channel then goes as

$$
\Gamma_{r} \approx a^{2} \frac{m_{r}^{3}}{192 \pi M_{P}^{2}},
$$


where the coupling constant $a \sim \mathcal{O}(1)$ depends on the type of radion, specific decay channel and the number of dimensions. $a$ is actually given in terms of the previously introduced coupling constants $\alpha, \beta$ and $\gamma$ (see section three). The life time of the radion is then given by

$$
\tau_{r} \approx 1.2 \times 10^{7} \mathrm{yr}\left(\frac{\mathrm{GeV}}{m_{r}}\right)^{3} .
$$

It is illustrative to see that a radion of mass $m_{r} \approx 30 \mathrm{MeV}$ has a life time $\tau_{r} \approx 10^{11} \mathrm{yr}$, which is already about the age of the universe. Lighter radions would then be effectively stable, so they might be good candidates for the cold dark matter. For heavier masses the radions would decay into visible channels (photons and leptons) which might leave a detectable trace. For instance, for masses just around few $\mathrm{MeV}$, radions can be produced not only in the early universe but also in supernova cores. Its later decay would inject hard photons of $\mathrm{MeV}$ energy at present time contributing to the diffuse gamma ray background flux, however, it is not too different from the one induced by KK graviton decays [10], and so, no new bounds on the fundamental scale can be obtained from this effect. In contrast, a radion mass about a few $\mathrm{TeV}$ would decay into energetic SM particles just right at the $\mathrm{BBN}$ era $(\sim 1-10 \mathrm{sec})$, which may photo-disintegrate primordial nuclei. Obviously, a radion mass within MeVs up to just below TeVs should decay somewhere within BBN and present time. Radions with larger masses than $10 \mathrm{TeV}$, or so, would decay fast enough as to wash out any primordial radion abundance. They could potentially reheat the universe with the SM degrees of freedom.

\section{RADION DRIVEN INFLATION}

The two radions evolve dynamically. It could be interesting to check whether by choosing an appropriate radion potential, one can drive inflation or not. Let us consider type I potentials for $\sigma_{v}, \sigma_{\perp}$. As already mentioned, irrespective of the masses $m_{v}, m_{\perp}$, the initial vevs are large in 4 dimensions, e.g. $\left|\sigma_{v}(0)\right| \sim\left|\sigma_{\perp}(0)\right| \sim \mathcal{O}\left(10 M_{p}\right)$. At such large vevs the initial conditions are equivalent to chaotic initial conditions, and if there is no other source for cosmological constant, then inflation should take place along the line of double inflation [40]

$$
V \sim \frac{1}{2} m_{v}^{2} \sigma_{v}^{2}+\frac{1}{2} m_{\perp}^{2} \sigma_{\perp}^{2} .
$$


Both the components give rise to the Hubble expansion, for which we obtain $H \approx$ $10 \max \left[m_{v}, m_{\perp}\right]$. Using the mass window in Eq.(5.4), we see that $H_{\text {inf }}$ could lie between $\mathcal{O}\left(10^{-2} \mathrm{eV}\right) \leq H_{\text {inf }} \leq \mathcal{O}\left(M_{*}\right)$. Inflation will terminate when slow roll conditions are no longer valid, which happens in the chaotic model when $\sigma_{v}, \sigma_{\perp} \sim \sqrt{2} M_{p}$.

During inflation both the fields generate quantum fluctuations which leave the horizon. We can estimate the amplitude for their density perturbations as

$$
\frac{\delta \rho}{\rho} \approx \frac{\max \left[m_{v}, m_{\perp}\right]}{M_{p}}<\frac{M_{*}}{M_{P}} .
$$

Note that the present constraint on the amplitude for the primordial density perturbations is given by one part in $10^{5}$, this is the COBE normalization verified by recent experiment such as WMAP [35, 36]. Unfortunately neither $m_{v}$ nor $m_{\perp}$ is sufficiently large to generate the right amplitude for the density perturbations in this radions driven inflation, as the bound on the RHS of the last equation indicates.

An interesting possibility may arise if the total radion potential is given by a combination, such as

$$
V \sim m_{v}^{2} M_{p}^{2}\left(1-e^{\alpha_{v} \sigma_{v} / M_{p}}\right)^{2}+\frac{1}{2} m_{\perp}^{2} \sigma_{\perp}^{2}
$$

where we assume a particular scenario with $m_{v} \sim \mathcal{O}(1 \mathrm{TeV})$, assuming for instance that there is some bulk physics which gives rise to the origin of volume stabilization, for e.g. flux quantization [41]. Let us also assume for the time being $m_{\perp} \ll m_{v}$. Note that here we get inflation from $\sigma_{v}$ radion, because the potential has a flat direction for large and negative vevs, e.g. $\sigma_{v}(0) \sim-\mathcal{O}\left(10 M_{p}\right)$. Clearly for this case the exponential part on the potential is negligible, and the cosmological constant dominates, such that $V \sim m_{v}^{2} M_{P}^{2}$. The Hubble expansion during inflation is given by $H_{\text {inf }} \approx m_{v}$. Again it is easy to verify that the adiabatic density perturbations produced by $\sigma_{v}$ during inflation will be way too small compared to $10^{-5}$, therefore one has to seek alternative ways of generating density perturbations. One such possibility is described below.

Whenever there are more than one fields involved during inflation, the quantum fluctuations can be of two types, pure adiabatic fluctuations, which tells us about the perturbations along the final trajectory of the fields, and second one is the isocurvature fluctuations which designates the perturbations along the orthogonal direction of the trajectory. In general isocurvature fluctuations arise due to difference in pressure fluctuations and most importantly isocurvature fluctuations feed the adiabatic fluctuations outside the horizon. 
In our case $\sigma_{\perp}$ being light compared to the Hubble expansion during inflation will also have homogeneous fluctuations. These fluctuations are actually isocurvature in nature.

The amplitude for the isocurvature fluctuations generated by $\sigma_{\perp}$ during inflation can be given by [42]

$$
\mathcal{P}_{\sigma_{\perp}}^{1 / 2} \sim \frac{H}{2 \pi \sigma_{\perp}} \sim \frac{m_{v}}{2 \pi \sigma_{\perp}} .
$$

In order to obtain the right amplitude for the density perturbations, $\sigma_{\perp} \sim 10^{5} \times m_{v}$. If $m_{v} \sim 1 \mathrm{TeV}$, then the vev of $\sigma_{\perp} \sim 10^{8} \mathrm{GeV}$. However since the mass of $\sigma_{\perp}$ is very light and the initial amplitude, i.e. $\left|\sigma_{\perp}\right| \sim \mathcal{O}\left(10 M_{p}\right)$ is quite large, it will be extremely hard for $\sigma_{\perp}$ to roll down from $M_{p} \sim 10^{18} \mathrm{GeV}$ to the amplitude $10^{8} \mathrm{GeV}$. Especially note that during inflation the radions are both slow rolling. Therefore the evolution of $\sigma_{\perp}$ can be written as

$$
3 H \dot{\sigma}_{\perp} \approx-m_{\perp}^{2} \sigma_{\perp} .
$$

Integrating this out we obtain

$$
\sigma_{\perp}(t) \approx \sigma_{\perp}(0) e^{-\left(m_{\perp}^{2} / 3 H\right) t} .
$$

For $m_{\perp} \ll H$, it would take many e-foldings of inflation before the field could be settled down to its minimum. Anther way of saying this is that in order to obtain $\sigma_{\perp} \sim 10^{8} \mathrm{GeV}$ from the initial value $10 M_{p}$, it would require between $76 \leq \mathcal{N}_{e} \leq 10^{16}$, for $1 \mathrm{TeV} \geq m_{\perp} \geq 10^{-3} \mathrm{eV}$, respectively, where $\mathcal{N}_{e}$ is the number of e-foldings. There is certainly a possibility that one of the radions could drive inflation while the other generates adequate density perturbations. If this can be realized then this will be the simplest model to be considered.

\section{A. Reheating \& moduli problem}

Let us briefly discuss the reheating temperature due to the decay of radions. During coherent oscillations, the radions can decay into SM degrees of freedom. The couplings among radions and brane SM fields have been discussed in section 3. By looking at those expressions it is easy to see that the presence of a non trivial radion vacuum would have two effects: it can change the normalization of the wave functions through the global radion couplings to the kinetic terms; and it can affect the gauge and Yukawa couplings as well as the potential terms. Nevertheless the overall radion couplings remain Planck suppressed. Therefore for our naive estimations one may still use the results given in section 6 . 
Radion decay products must hadronize before BBN [43]. If however the radion decays much later than BBN then there will be two problems: (1) hadronizing the universe with SM relativistic degrees of freedom may not be complete, and (2) even if thermalization is completed well before BBN, the radion decay products with hard momentum can eventually destroy the light elements by reheating the universe.

Using Eq. (6.1) the perturbative reheating temperature can be estimated by

$$
T_{r} \approx 0.1 \sqrt{M_{P} \Gamma_{r}}=1.28 \times 10^{-7} \mathrm{GeV} \cdot\left(\frac{m_{v}}{\mathrm{TeV}}\right)^{3 / 2}
$$

Therefore, from our naive analysis, it seems that the only solution to this problem is that the radion $\sigma_{v}$ must be heavier than about $500 \mathrm{TeV}$. Indeed for a mass at such value the reheating temperature is just about $T_{r} \sim 1 \mathrm{MeV}$. This also forbids the possibility of having a $\mathrm{TeV}$ fundamental scale in this scenario ${ }^{1}$.

Let us discuss the associated moduli problem. In the above scenario a moduli problem will occur due to the coherent oscillations of $\sigma_{\perp}$. Note that $\sigma_{\perp}$ oscillates with a frequency $m_{\perp}$ when $H \sim m_{\perp}$. Depending on the initial amplitude of the coherent oscillations of $\sigma_{\perp}$ there can be adverse affects on cosmology. The energy density stored in $\rho_{\sigma_{\perp}} \approx m_{\perp}^{2} \sigma_{\perp}^{2}$ will redshift as in a matter dominated era and will eventually dominate the relativistic bath already created by the decay products of $\sigma_{v}$, provided $\rho_{\sigma} \approx H^{2} M_{p}^{2}$. However this requires the amplitude of $\sigma_{\perp}$ oscillations to be as large as $\sigma_{\perp}(0) \sim M_{p}$. On the other hand, if the initial amplitude of the oscillations are sufficiently low, e.g. $\sigma_{\perp} \leq M_{p}$ then it is certainly possible to avoid the radion dominance problem. This may occur if there is a large enough e-foldings of inflation.

\section{BRANE INFLATION AND ITS CONSEQUENCES}

In this section we consider a possibility where the inflaton potential arises from brane physics. The scalar field action is given by Eq. (3.6). We suppose that a hypothetical scalar field $\chi$ is living on the $4+n$ dimensional brane and its potential is given by $V(\chi)$. The total action with the radions is given by

\footnotetext{
${ }^{1}$ Interesting possibility may arise if the reheating temperature is asserted to be the Hagedorn temperature, which comes out to be close to the mass of the radions, i.e. $T_{r} \sim 0.74 \times m_{v} \leq \mathrm{TeV}$, see 44 .
} 


$$
\begin{aligned}
S=\int d^{4} x \sqrt{-g}\left[\frac{1}{\kappa^{2}} R+\frac{1}{2}\left(\partial_{\mu} \sigma_{v}\right)^{2}\right. & +\frac{1}{2}\left(\partial_{\mu} \sigma_{\perp}\right)^{2}+\frac{1}{2} e^{-\left(\beta \sigma_{v}+\gamma \sigma_{\perp}\right) / M_{p}}\left(\partial_{\mu} \chi\right)^{2} \\
& \left.-e^{-\left((\beta+\alpha) \sigma_{v}+\gamma \sigma_{\perp}\right) / M_{p}} V(\chi)-U\left(\sigma_{v} \sigma_{\perp}\right)\right] .
\end{aligned}
$$

The natural scale for the effective $V(\chi)$ in 4 dimensions is

$$
V(\chi) \lesssim\left(M_{*} r\right)^{n} M_{*}^{4} \simeq 10^{n} M_{*}^{4}
$$

The numerical factor on the RHS arises when the compactification scale for $n$ extra spatial dimensions is $M_{c} \sim 10^{-1} M_{*}$. Initial phase of inflation is governed by the exponential part $\exp \left[-\left((\beta+\alpha) \sigma_{v}+\gamma \sigma_{\perp}\right) / M_{p}\right] V(\chi)$, since $\sigma_{v}(0), \sigma_{\perp}(0) \simeq-\mathcal{O}\left(10 M_{p}\right)$, which exponentially enhances that contribution.

In slow roll approximations, where we neglect the higher order time derivatives and we assume that the friction term proportional to the Hubble expansion dominates, the equations of motion for the fields and the Hubble parameter simplify a lot, and one gets

$$
\begin{aligned}
3 H \dot{\sigma}_{v} & \approx \frac{\beta+\alpha}{M_{p}} e^{-\left((\beta+\alpha) \sigma_{v}+\gamma \sigma_{\perp}\right) / M_{p}} V(\chi) \\
3 H \dot{\sigma}_{\perp} & \approx \frac{\gamma}{M_{p}} e^{-\left((\beta+\alpha) \sigma_{v}+\gamma \sigma_{\perp}\right) / M_{p}} V(\chi) \\
3 H \dot{\chi} & \approx-e^{-\left(\alpha \sigma_{v}\right) / M_{p}} \frac{\partial V}{\partial \chi} \\
H^{2} & \approx \frac{1}{3 M_{p}^{2}}\left[e^{-\left((\beta+\alpha) \sigma_{v}+\gamma \sigma_{\perp}\right) / M_{p}} V(\chi)+U\left(\sigma_{v}, \sigma_{\perp}\right)\right] .
\end{aligned}
$$

Let us assume that during inflation $\exp \left[-\left((\beta+\alpha) \sigma_{v}+\gamma \sigma_{\perp}\right) / M_{p}\right] V(\chi) \gg U\left(\sigma_{v}, \sigma_{\perp}\right)$. We can also assume that $V(\chi)$ piece is dominated by a false vacuum and it is merely constant during inflation. However, note that $\sigma_{v}$ and $\sigma_{\perp}$ are present in the exponential part of the potential. The rolling of the radions will govern the initial phase of inflation in such a scenario. This is an example of assisted inflation, for which the scale factor follows [45]

$$
a(t) \propto t^{\kappa}, \quad \kappa=\frac{2}{(\alpha+\beta)^{2}+\gamma^{2}} .
$$

Here we have assumed that $V(\chi)$ is a flat potential, which means that $\chi$ evolution is slower compared to $\sigma_{v}, \sigma_{\perp}$. In our case the power law inflation lasts as long as $\left|\sigma_{v}\right|,\left|\sigma_{\perp}\right| \sim M_{p}$. Once the vevs of both the radions become smaller than $M_{p}$, then effective masses for the 
radions become

$$
\begin{aligned}
& m_{v e f f}^{2} \approx \frac{\partial^{2} U}{\partial \sigma_{v}^{2}}+(\beta+\alpha)^{2} \frac{V(\chi)}{M_{p}^{2}} \\
& m_{\perp e f f}^{2} \approx \frac{\partial^{2} U}{\partial \sigma_{\perp}^{2}}+\gamma^{2} \frac{V(\chi)}{M_{p}^{2}}
\end{aligned}
$$

If $V(\chi) / M_{p}^{2}$ were greater than the bare masses for the radions, then an effective radion mass would become of order $H_{\text {inf }} \sim V(\chi) / M_{p}^{2}$. However, since $V(\chi) \lesssim\left(M_{*} r\right)^{n} M_{*}^{4}$, this means $H_{\text {inf }}$ is many orders of magnitude smaller than $M_{*}$. For instance, for $M_{*} \sim 1 \mathrm{TeV}$ and $M_{*} r \sim 10$, we obtain an inflationary scale of order $H_{\text {inf }} \lesssim 10^{n} \times 10^{-2} \mathrm{eV}$ !. This also implies

that the radions potential, $U$, should be extremely flat. Certainly $H_{\text {inf }}$ improves for larger vales of $n$ and $M_{*}$, but still it is hard to push the scale of inflation up to $\mathcal{O}(\mathrm{TeV})$. Such low scale of inflation has many problems to produce successful baryogenesis and BBN [21].

On the other hand if radion masses win over inflaton scale in Eqs. (8.8) and (8.9), then inflation ends right after $\sigma_{v}, \sigma_{\perp} \sim M_{p}$. The radions start oscillations around their minimum and eventually decay into SM degrees of freedom. Note that the radion oscillations are large with the amplitudes of the order of $M_{p}$. The late domination of radion can occur if there is a hierarchy in radion masses. The lighter one, if it does not decay completely, can cause a problem similar to the moduli problem as discussed before.

\section{BULK INFLATION}

As a final possibility we investigate the bulk driven inflation. We assume that inflation arises from the bulk physics, e.g. a scalar field $\phi$ living in $4+d$ dimensions, such that the action is given by

$$
S=\int d^{4} x \sqrt{-g}\left[\frac{1}{2}\left(\partial_{\mu} \sigma_{v}\right)^{2}+\frac{1}{2}\left(\partial_{\mu} \sigma_{\perp}\right)^{2}+\frac{1}{2}\left(\partial_{\mu} \phi\right)^{2}-e^{-\alpha \sigma_{v} / M_{p}} V(\phi)-U\left(\sigma_{v}, \sigma_{\perp}\right)\right] .
$$

The four dimensional action is much simpler in this case. Note that after dimensional reduction the effective potential is $V(\phi) \lesssim M_{*}^{2} M_{p}^{2}$, which can be relatively large compared to the former scenario where we assumed that inflaton potential as originating from the brane physics. Let us discuss this scenario in some detail.

First of all note that the radion $\sigma_{v}$ only couples to the inflaton potential and not $\sigma_{\perp}$. Therefore $\sigma_{\perp}$ does not get any effective mass correction through the inflaton potential, while 
the volume modulus does. As we shall see this will play an interesting role in ameliorating the moduli, or late domination of radion in thermal history of the universe.

\section{A. Inflationary dynamics}

The equations of motion for the above action are given by

$$
\begin{aligned}
& \ddot{\sigma}_{v}+3 H \dot{\sigma}_{v}+\frac{\alpha}{M_{p}} e^{\alpha \sigma_{v} / M_{p}} V(\phi)-\frac{\partial U}{\partial \sigma_{v}}=0, \\
& \ddot{\sigma}_{\perp}+3 H \dot{\sigma}_{\perp}-\frac{\partial U}{\partial \sigma_{\perp}}=0, \\
& \ddot{\phi}+3 H \dot{\phi}-e^{-\alpha \sigma_{v} / M_{p}} \frac{\partial V}{\partial \phi}=0, \\
& H^{2}=\frac{1}{3 M_{p}^{2}}\left[\frac{\left(\dot{\sigma}_{v}\right)^{2}}{2}+\frac{\left(\dot{\sigma}_{\perp}\right)^{2}}{2}+\frac{(\dot{\phi})^{2}}{2}+e^{-\alpha \sigma_{v} / M_{p}} V(\phi)+U\right] .
\end{aligned}
$$

In this particular scenario there are two phases of inflation possible. Initial phase of inflation is driven by the exponential part of the potential provided there is flat potential for $V(\phi)$. This phase is essentially driven by radion, and the subsequent phase of inflation is governed by $V(\phi)$ with a Hubble parameter $H \lesssim M_{*}$. During inflation the slow roll equations are given to a good approximation by

$$
\begin{aligned}
3 H \dot{\sigma}_{v} & \approx-\frac{\alpha}{M_{p}} e^{\alpha \sigma_{v} / M_{p}} V(\phi)+\frac{\partial U}{\partial \sigma_{v}} \\
3 H \dot{\sigma}_{\perp} & \approx \frac{\partial U}{\partial \sigma_{v}} \\
3 H \dot{\phi} & \approx e^{-\alpha \sigma_{v} / M_{p}} \frac{\partial V}{\partial \phi}, \\
H^{2} & \approx \frac{1}{3 M_{p}^{2}}\left[e^{-\alpha \sigma_{v} / M_{p}} V(\phi)+U\right] .
\end{aligned}
$$

The scale factor $a(t)$ follows

$$
a(t) \propto t^{(n+p+2) / n+p}
$$

by assuming that $V(\phi) \gg U$ and $\phi$ evolution is extremely slow. Note that again we obtain

a power law inflation. The power law inflation does not last for ever. Once $\sigma_{v} \sim-M_{p}$, inflation is not supported along $\sigma_{v}$ direction, rather inflation is supported by the inflaton potential $V(\phi)$. Also note that when the vev of $\sigma_{v}$ becomes less than $M_{p}$, we can expand the exponential term $\exp \left(-\alpha \sigma_{v} / M_{p}\right)$. The effective mass for $\sigma_{v}$ can be expressed as

$$
m_{\sigma_{v} \text { eff }}^{2} \approx \alpha \frac{V(\chi)}{M_{p}^{2}}+\frac{\partial^{2} U}{\partial \sigma_{v}^{2}} \simeq 3 \alpha H_{i n f}^{2} .
$$


Where we have assumed $H_{\text {inf }} \approx V(\phi) / M_{p}^{2}$, irrespective of the form of $V(\phi)$, provided of course that $V(\phi)$ is sufficiently flat to support inflation. Further note that if the bare mass for $\sigma_{v}$ is small compared to $H_{\text {inf }} \sim M_{*}$, then the Hubble induced mass term will dominate. Once Hubble induced mass term is switched on the radion $\sigma_{v}$ will follow the evolution

$$
\sigma_{v}(t) \sim \sigma_{v i} e^{-\left(m_{e f f}^{2} / 3 H\right) t}
$$

where $\sigma_{v}$ is the initial amplitude which is of order $M_{p}$. The volume modulus therefore settles in its local minimum within one Hubble time. Note, however, that the global minimum for $\sigma_{v}$ could be displaced during inflation. If such a displacement is large enough another phase of inflation could take place. That generally happens when the radion potential is quite flat [46]. We will discuss this issue in the next subsection.

Further note that although the amplitude of $\sigma_{v}$ can be reduced considerably during inflation, the same can not be true for the shape mode, $\sigma_{\perp}$. The other radion does not obtain a Hubble induced mass correction during inflation, because it does not couple to $\phi$ field at all at classical level. Therefore the amplitude of $\sigma_{\perp}$ may not damp as much as we wish. Depending on its mass and its vev it might be possible that $\sigma_{\perp}$ could be responsible for generating adiabatic density perturbations, which we will discuss later on.

As noted earlier, we could easily get two subsequent bouts of inflation; initially driven by the radion $\sigma_{v}$ and then by the inflaton field in the bulk. However number of e-foldings required for the structure formation depends on the exact thermal history of the universe. Usually the number of e-foldings is much less than 60 if the scale of inflation is low. Especially if the scale of inflation is as low as $H_{\text {inf }} \approx M_{*}$, it was already shown in [23, 24] that the number of e-foldings required for structure formation would be 43 , provided the universe reheats by $T_{r h} \sim 10-100 \mathrm{MeV}$. Therefore it is only the last 43 e-foldings of inflation which are important for the purpose of density perturbations.

So far we have not specified the form of $V(\phi)$. However we have noticed that the upper bound on bulk inflation energy density is $\sim M_{*}^{2} M_{\mathrm{p}}^{2}$. Therefore the second bout of inflation, which is mainly driven by the bulk field, $\phi$, the scale of inflation, $H_{\text {inf }} \sim M_{*}$, which for $M_{*} \sim \mathcal{O}(\mathrm{TeV})$, is sufficiently low. It is easy to demonstrate that a single bulk inflaton model does not produce adequate density perturbations (see for instance the discussions in Ref. [21]), whereas a two field bulk inflation model, where inflation ends via a phase transition similar to hybrid inflation scenario, can generate the COBE normalization [22]. 
The effective four dimensional potential for two fields is given by [22]

$$
V(\phi, N) \equiv\left(\frac{M_{\mathrm{p}}}{M_{*}}\right)^{2} \lambda^{2} N_{0}^{4}+\frac{\lambda^{2}}{4}\left(\frac{M_{*}}{M_{\mathrm{p}}}\right)^{2} N^{4}-\lambda^{2} N_{0}^{2} N^{2}+g^{2}\left(\frac{M_{*}}{M_{\mathrm{p}}}\right)^{2} \phi^{2} N^{2}+\frac{1}{2} m_{\phi}^{2} \phi^{2},
$$

where $\phi, N$ are zero modes of the bulk fields. Note that the four dimensional couplings are Planck suppressed, which comes out naturally by integrating out the extra $d$ dimensions. The naturalness condition requires the bulk couplings $\lambda, g \sim \mathcal{O}(1)$, and $N_{0} \sim M_{*}$. Inflation in this scenario is supported by the false vacuum $\lambda^{2} M_{*}^{2} M_{p}^{2}$, because during inflation $N$ settles in its local minimum, $N=0$, and the rolling of $\phi$ field gives rise to the phase transition. However one has to ensure that the false vacuum contribution dominates over $(1 / 2) m_{\phi}^{2} \phi^{2}$ term. In this respect it is plausible to give a small mass term for the $\phi$ field, $m_{\phi} \ll M_{*}$. Note that in this scenario inflation ends when

$$
\phi_{c}=\frac{\lambda}{g}\left(\frac{N_{0} M_{\mathrm{p}}}{M_{*}}\right) .
$$

Note, if $\lambda \sim g$, and, $N_{0} \sim M_{*}$, we automatically get $\phi_{\mathrm{c}} \sim M_{\mathrm{p}}$.

This potential was further studied numerically in Ref. [24], and it was shown that in this scenario the phase transition which ends inflation happens extremely slowly. In the most natural case, $N_{0} \sim M_{*}, \lambda, g \sim \mathcal{O}(1)$, and $m_{\phi} \leq 0.1 M_{*}$, it is possible to have extremely large number of e-foldings of order, $\mathcal{N}_{e} \sim \mathcal{O}\left(10^{6}\right)$.

The occurrence of large number of e-foldings can be understood due to the fact that the shape of the potential along the $N$ direction is extremely flat due to extremely small coupling. The field $N$ rolls down extremely slowly and spends a considerable time near the top of the potential before rolling down to its global minimum $N \sim \sqrt{2} M_{*}$, while the $\phi$ field rolls slowly away from $\phi_{c}$ towards its global minimum $\phi=0$. It can be shown that inflation ends in this picture when [24]

$$
2 g^{2}\left(\frac{M_{*}}{M_{\mathrm{P}}}\right)^{2} N^{2}(t) \sim m_{\phi}^{2}
$$

The phase transition could be made rapid, or the number of e-foldings during the phase transition can be made small, provided there is a hierarchy $m_{\phi}<<N_{0}<M_{*}$.

\section{B. The Radion Problem}

Let us consider once more the potential of the radion-inflaton system,

$$
V\left(\sigma_{v}, \phi\right)=e^{-\alpha \sigma_{v} / M_{p}} V(\phi)+U\left(\sigma_{v}\right)
$$


Even though the global minimum do correspond to $\sigma_{v}=0$, it is easy to see that during the phase of inflation driven by the inflaton potential the radion is trapped in a a false (effective) minimum. Indeed, if one takes the inflaton potential as effectively constant, the minimization condition for the total radion potential reads

$$
\frac{\partial U\left(\sigma_{v}\right)}{\partial \sigma_{v}}=\frac{\alpha}{M_{P}} e^{-\alpha \sigma_{v} / M_{p}} V(\phi) .
$$

Clearly, $\sigma_{v}=0$ is not longer a solution, unless $V(\phi)$ were null. Thus, the minimum for the radion is displaced [46] to a position which strongly depends on the actual form of the radion potential. We should notice, however, that this effective minimum is always located on the positive range of $\sigma_{v}$ values, which means that within a Hubble time the bulk has grown larger than its expected value (see the definition of the bulk radion in Eq. (2.9)). This can be understood by noticing that the global minimum of $\sigma_{v}$ is at zero, and the radion is originally coming down the potential from negative values, therefore, only for $\sigma_{v}>0$ one could have a positive slope for $U\left(\sigma_{v}\right)$, as it is needed to match the RHS of Eq. (9.17). Moreover, this equation indicates that the effective minimum is located at a $\sigma_{v}$ value where the slope of the radion potential catches up with a now suppressed inflaton potential.

To clarify these points, let us take for instance the potential $U\left(\sigma_{v}\right)=\frac{1}{2} m^{2} \sigma_{v}^{2}$, where the radion mass is assumed to be lighter than the inflaton potential scale, $M_{I}$. Here $M_{I}$ is taken to be the inflation scale as given by the inflaton potential alone (i.e. we use $V(\phi)=M_{P}^{2} M_{I}^{2}$ ). In Figure 1 we have plotted the total radion potential for a particular case where $\alpha M_{I} / m=$ 10. There we have also depicted each term on the potential. Now one can easily sees why and where the minimum gets displaced. First of all the inflaton part of the potential drops very fast due to the exponential suppression of a growing radion, however, this is not fast enough as to catch with the radion potential which, being quite flat, grows much more slowly. Next point is that the exponential has no minimum, so the minimum appears only due to $U\left(\sigma_{v}\right)$, and therefore, such a minimum should be close to the $\sigma_{v}$ value where both the potentials are almost equally relevant. Unfortunately, for the case we are considering, this happens when $\sigma_{v}$ is about three times larger than $M_{p}$. This effect is more dramatic when the hierarchy among $m$ and $H_{I}$ is larger. In such a case the inflaton potential in our picture is shifted to the right, which means that the minimum should be even at a larger $\sigma_{v}$ value.

This is troublesome for our previous picture of inflation: it means that, although the inflaton is slow rolling, its contribution to the Hubble expansion is being affected by the fast 


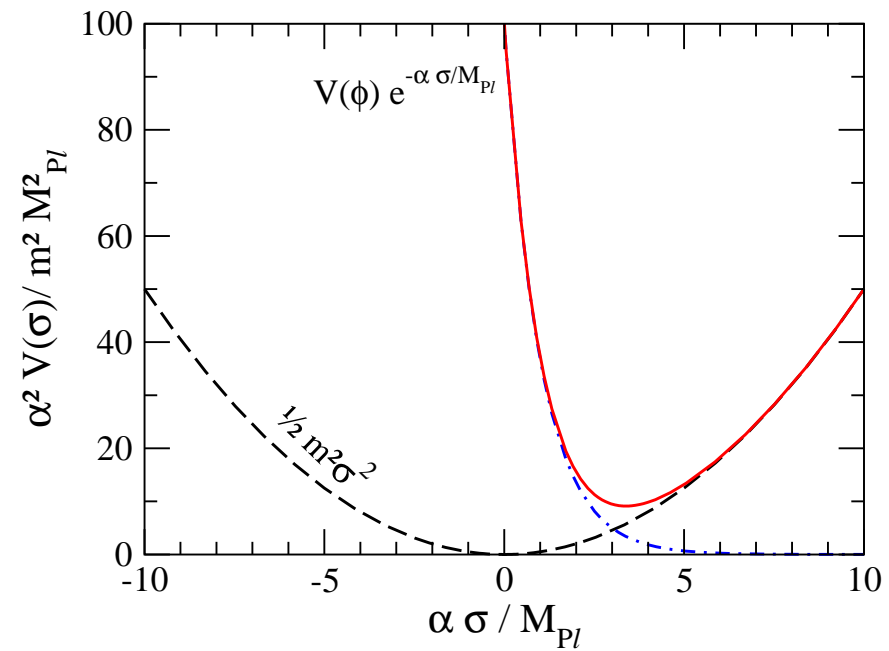

FIG. 1: Total radion potential for $\alpha M_{I} / m=10$ (continuous curve). Dashed curve corresponds to the radion mass potential whereas the dash-dotted curve plots the inflaton-radion coupling term. Notice the minimum of the total potential occurs close to the point where both terms are of same order.

evolution of the exponential suppression that comes with a growing radion. Once both the terms in the potential are comparable we cannot neglect the radion contribution to inflation anymore, which means that a third phase of inflation would take place. In this new phase both fields contribute to expansion, however, now Hubble scale would be much smaller than $M_{I}$ by some orders of magnitude. In fact, by using Eq. (9.17), we find that the equation for the minimum satisfies

$$
m^{2} \sigma_{v}=\frac{\alpha}{M_{P}} e^{-\alpha \sigma_{v} / M_{p}} V(\phi) .
$$

Using this expression one can evaluate the whole potential at the effective minimum and we obtain [46]

$$
V\left(\sigma_{v}\right)=m^{2} \sigma_{v}\left[\frac{1}{2} \sigma_{v}+\frac{M_{P}}{\alpha}\right] .
$$

In other words, the scale of inflation should now be $H_{\text {inf }} \sim m$.

The system will remain in the false vacuum as long as the inflaton remains constant. Inflaton was, nevertheless, slow rolling right before the radion field settles down to the false vacuum. As inflation proceeds, the inflaton slowly takes smaller vevs, as does the energy density residing in its potential. For small values of the scale of the $V(\phi)$ potential, $M_{I}$, the minimum gets shifted to smaller values of $\sigma_{v}$. It would look like if the radion were slowly rolling down an effective potential given by Eq. (9.18). By looking at the RHS of 


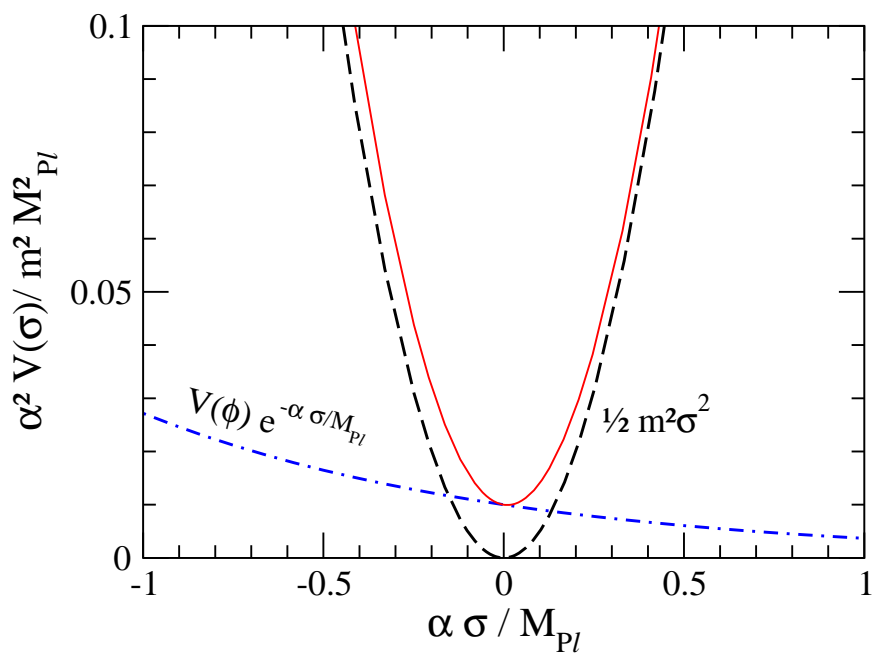

FIG. 2: Same as before but now taking $\alpha M_{I} / m=0.1$. Notice that here the minimum is consistent with $\sigma_{v}=0$.

Eq. (9.9) we find that the exponential suppression of the inflaton potential does contribute for a very slow evolution of the inflaton field, which behaves as if it were effectively frozen. All these would result in a large period of inflation, that may last plenty of e-foldings. As inflation continues in 4D, the radion rolls down and the extra volume now shrinks. It ends only when the inflaton leaves the slow rolling phase, by then it is very likely that the radion would still have a large, almost Planckian vev. Therefore, just after inflation ends the radion would again start oscillating with an amplitude of order of the Planck mass. Therefore bulk inflation may not help to completely solve the radion problem.

On the other hand, this problem does not appear if the radion mass were larger than the scale of the inflaton potential. Figure 2 plots the radion potential when $\alpha M_{I} / m=0.1$. Now the whole picture changes dramatically, the radion mass dominates on the dynamics of expansion in a first phase. If the mass is large enough the radion quickly goes down the potential whereas the inflaton remains frozen. At some point, when the radion amplitude is quite small in the picture, the inflaton takes over and drives the radion towards the effective minimum, which is nicely just around $\sigma_{v}=0$. From there on the evolution would be dominated by the inflaton potential and our discussion in the previous subsection applies. 


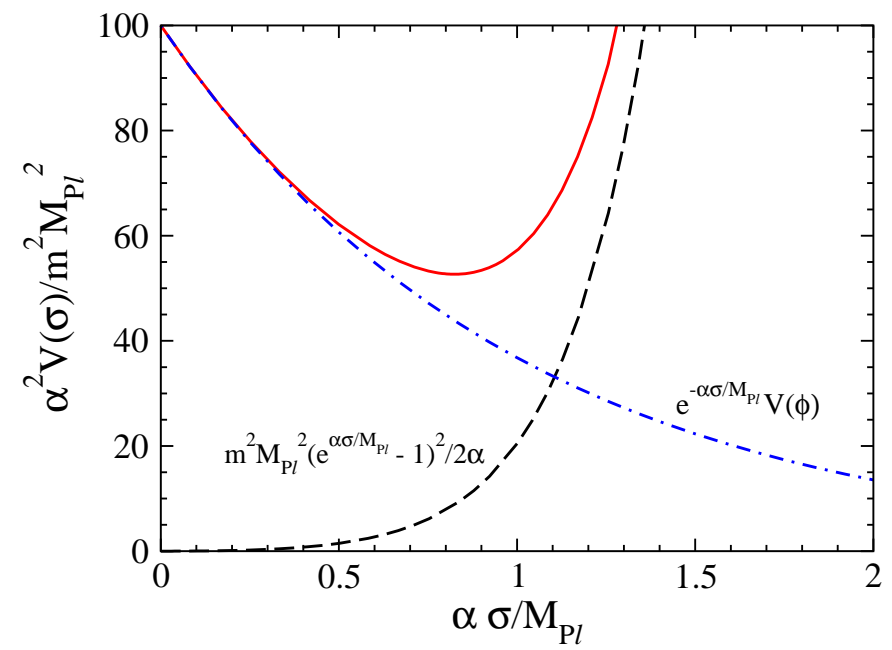

FIG. 3: The total radion potential as seen when the radion stabilization potential $U\left(\sigma_{v}\right)$ becomes rather steep for positive values of $\sigma_{v}$. Here we have used $\alpha M_{I} / m=10$. Notice that now the false minimum is closer to zero than before.

\section{A potential resolution to the radion problem}

Finally, let us consider what happens if the radion stabilization potential were similar to the one described in Eq. (5.3):

$$
U\left(\sigma_{v}\right) \sim m_{v}^{2} M_{p}^{2}\left(1-e^{\alpha_{v} \sigma_{v} / M_{p}}\right)^{2}
$$

We use this potential and plot the total radion potential in Figure 3 for the same hierarchy of parameters used in Figure 1. Now, the stabilization potential grows exponentially for positive $\sigma_{v}$ values and then catches up with the inflaton potential not far from $\sigma_{v}=0.5 M_{P}$. In contrast to what happened in the case depicted in Figure 1, now the bulk volume is not allowed to grow too much beyond the stabilized value and this ameliorates the radion problem. Notice also that at the effective minimum the total potential is still dominated by the inflaton, therefore, it gives the features we were taking as granted in the previous subsections. Once the radion is settled at the effective minimum expansion would be driven only by the inflaton. This kind of potentials gives an idea of what one would desire for the actual stabilization potential. Therefore we conclude in a cheering note that indeed if the correct radion potential is found we would be able to stabilize the radion to its true minimum during inflation, and thereby solve the radion problem. 


\section{Reheating}

It is believed that the total energy density of the inflaton is transferred into radiation. The minimal requirement of cosmology is to have a thermal bath with a temperature more than $\mathcal{O}(1) \mathrm{MeV}$ in order to preserve the successes of BBN. The final reheat temperature depends on the decay rates $\Gamma_{\phi, N}$ of the oscillating field

$$
T_{\mathrm{r}} \sim 0.1 \sqrt{\left(\Gamma_{\phi}+\Gamma_{N}\right) M_{\mathrm{p}}} .
$$

Note that in our model the bulk fields couple to effective 4 dimensions via Planck suppressed interactions with the SM fields on the observable brane.

However the zero mode inflaton can reheat the brane and also the bulk. This possibility was already addressed in Ref. [22] for the case of gravitons. From Eq. (9.13) we found that

both the fields obtain effective mass terms $\bar{m}_{\phi} \sim \bar{m}_{N} \propto \sqrt{2} \lambda N_{0}$, if we assume $\lambda=g$. Setting $N_{0} \sim \mathcal{O}(\mathrm{TeV})$, then both $\phi$ and $N$ are kinematically allowed to decay into SM Higgs field, $h$. The decay rate can be estimated as follows [22]

$$
\Gamma_{\phi, N \rightarrow h h} \sim \frac{f^{2} M_{*}^{4}}{32 \pi M_{\mathrm{p}}^{2} m_{\phi, N}},
$$

where $f$ is the coupling constant that we take of order one. If $\bar{m}_{\phi} \sim \bar{m}_{N} \approx 0.1 M_{*}$, then the reheat temperature is $T_{\mathrm{r}} \leq 100 \mathrm{MeV}$, for $M_{*} \sim 100 \mathrm{TeV}$. Note that such a low reheat temperatures is a generic prediction if the inflaton field is living in the bulk.

The next point is concerning the possible production of KK gravitons by the inflaton. The KK gravitons can be directly produced from the decay of KK modes of $\phi$ and $N$, through the channel $\phi_{n} \rightarrow \phi_{l} G_{n-l}$, where $n, l$ are the KK numbers, and $G$ is the KK graviton. On the other hand $\phi_{n}$ modes can be produced via collision processes, such as $\phi \phi \rightarrow \phi_{n} \phi_{-n}$, and similar reaction for $N$. The rate for exciting KK inflaton modes goes as

$$
\sigma_{\phi \phi \rightarrow \phi_{n} \phi_{-n}} \sim \lambda^{2} \frac{M_{*}^{2}}{M_{\mathrm{p}}^{4}}
$$

whereas the total decay rate of a KK scalar decaying via graviton emission is estimated as

$$
\Gamma_{\phi_{n}, t o t a l}=\sum_{\ell<n} \Gamma_{\phi_{n} \rightarrow \phi_{\ell} G_{n-\ell}} \sim \frac{m_{n}^{3}}{12 \pi M_{*}^{2}},
$$

where $m_{n}^{2}=\bar{m}_{\phi}^{2}+n^{2} / R^{2}$. Note that the $N N$ or $\phi \phi$ scattering rates for producing their KK counterparts are more Planck suppressed than the direct decay of $\phi, N$ to the brane fields, 
i.e. $\Gamma_{\phi \rightarrow h h} \gg \sigma_{\phi \phi \rightarrow \phi_{n} \phi_{-n}} \times M_{*}^{3}$; where we have taken the number density of modes $\sim M_{*}^{3}$. So, we find that the zero modes of $\phi$ and $N$ still prefer the Higgs as a final decay product. On the other hand, the excited KK inflaton or KK partners of other scalar fields present in the spectrum are extremely short lived. The heavier KK mode decays into the lighter KK modes plus gravitons, and eventually all the KK modes of the inflaton decay into the zero mode. In case where extra bulk fields are present the reheating of the bulk can be naturally avoided, if either those modes are as heavy as the inflaton, or the effective inflaton couplings to the bulk fields are smaller than the inflaton-brane interactions.

\section{DENSITY PERTURBATIONS}

\section{A. Converting radion isocurvature fluctuations to adiabatic perturbations via ra-} dion dominance

We recognize that in a bulk inflation scenario the reheat temperature of the universe is small, $T_{r} \sim 1-10 \mathrm{MeV}$. The number of e-foldings required for the COBE normalization depends on thermal history of the universe after the end of inflation. For a low scale inflation and low reheating temperature the require number of e-foldings is small compared to the usual 60 e-foldings, it is roughly 43 e-foldings 23,24$]$. As we discussed in our earlier sections, the last 43 e-foldings can easily arise during the slow phase transition for natural parameters, e.g. $N_{0} \sim M_{*}, m_{\phi} \sim\left(10^{-1}-10^{-2}\right) M_{*}$. However as it was also pointed out in Ref. [24], the amplitude of the scalar density perturbations during the phase transition evolves gradually to become very small. Numerical studies suggest that for the above mentioned parameters the amplitude of the perturbations is way too small, e.g. $\mathcal{P}_{H}^{1 / 2}<10^{-15}$ [24]. The only way this situation could be ameliorated if there is a large hierarchy between the scales, e.g. $N_{0} \ll M_{*}, m_{\phi} \ll M_{*}$. The summary is that we do not gain on density perturbations by introducing two fields either. Nevertheless we should study other interesting options, such as radion induced adiabatic perturbations.

Note that in our case $\sigma_{v}$ can be dynamically stabilized during bulk inflation. However $\sigma_{\perp}$ cannot be dynamically driven to its minimum. As a consequence the actual dynamics of $\sigma_{\perp}$ can be treated independently. If $\sigma_{\perp}$ is sufficiently light compared to the Hubble expansion during inflation then quantum fluctuations along $\sigma_{\perp}$ direction generates isocurvature 
fluctuations, which could be transferred into adiabatic fluctuations when $\sigma_{\perp}$ decays while dominating the universe. However we have to keep in mind that $\sigma_{\perp}$ must decay before $\mathrm{BBN}$ and it must dominate the energy density of the universe while decaying. In case $\sigma_{\perp}$ dominates later on, then when it decays into SM degrees of freedom, it will inject significant entropy in the universe which will dilute the light nuclei created during BBN. Note that $\sigma_{\perp}$ decays only via Planck suppressed interactions. This leaves us with a narrow window for the mass range of $\sigma_{\perp}$, which has to be large, e.g $m_{\sigma_{\perp}}=500 \mathrm{TeV}$.

Important issue to raise is what should be the amplitude of isocurvature density perturbations produced by $\sigma_{\perp}$. Let us for the time being concentrate on flat radion potential $m_{\sigma_{\perp}}^{2} \sigma_{\perp}^{2}$. As we discussed in section seven, see Eq. (7.4), the amplitude of $\sigma_{\perp}$ during that last 43 efoldings of inflation must be $10^{5} \times M_{*}$. If we set an opportunistic value, $m_{\sigma_{\perp}}=5 \times 10^{5} \mathrm{GeV}$ and $M_{*} \sim 10^{6} \mathrm{GeV}$, then $\sigma_{\perp, \text { inf }} \sim 10^{11} \mathrm{GeV}$, in order to satisfy the COBE normalization. Obviously, depending on the number of extra spatial dimensions, if the initial vev of $\sigma_{\perp}$ at the onset of inflation is $\sigma_{\perp}(0) \sim-10 M_{p}$, see section six, then following Eq. (7.6) we can evaluate the approximate number of e-foldings required to bring down its vev to a value when the the interesting scalar perturbation modes are leaving the horizon. The number of e-foldings comes out to be roughly $\mathcal{N}_{e} \sim 3 \times 10^{5}$. Note that here we are required to relax $\mathrm{TeV}$ as a fundamental scale. The main bound comes from the radion mass which has to decay before $\mathrm{BBN}$ in order to convert isocurvature perturbations to adiabatic ones. Also note that radion has to dominate the energy density before decaying. This we will discuss below.

The conclusion is that if the fluctuations of $\sigma_{\perp}$ generates $\mathcal{P}_{\sigma_{\perp}}^{1 / 2} \sim 10^{-5}$, then there has to be a large number of e-foldings of inflation. In this respect any chaotic inflation model can do the job, it could either be a single field inflation, e.g. $V \sim m^{2} \phi^{2}$ with a large initial vev of $\phi \sim 10^{2}-10^{3} \times M_{p}$, or two field hybrid inflation model with a very slow phase transition, see Eq. (9.13).

The spectral index of the fluctuations can be determined by

$$
n-1 \equiv \frac{d \ln \mathcal{P}_{\sigma_{\perp}}}{d \ln k}=2 \frac{\dot{H}_{\mathrm{inf}}}{H_{\mathrm{inf}}^{2}}+\frac{2}{3} \frac{m_{\sigma_{\perp}}^{2}}{H_{\mathrm{inf}}^{2}} .
$$

If $\dot{H}_{\text {inf }} \ll H_{\text {inf }}^{2}$, which obviously depends on the bulk inflaton potential, then there is a slight dependence on the spectral index given by $n-1 \sim(2 / 3)\left(m_{\perp}^{2} / H_{\text {inf }}^{2}\right)$. On a generic ground one can say that if $m_{\sigma_{\perp}}<H_{\mathrm{inf}} \sim M_{*}$, then the spectral index is very close to Harrison Zeldovich 
case, e.g. $n \sim 1$. This is one of the signatures of radion induced fluctuations. Note that the energy density of the radion is always subdominant during inflation, e.g. $V>m_{\sigma}^{2} \sigma_{\perp}^{2}$.

However it is important that isocurvature perturbations stored in $\sigma_{\perp}$ must be converted into the adiabatic ones. This can happen only if $m_{\sigma_{\perp}}^{2} \sigma_{\perp}^{2}$ dominates at later stages, but before $\mathrm{BBN}$ era. Therefore, the radion must be responsible for finally reheating the universe. However note that in our case this can happen soon after the end of inflation, because when $m_{\sigma_{\perp}} \sim H(t)$, the radion starts oscillating with an amplitude which is not far from $10^{8} \mathrm{GeV}$. Note that radion like the inflaton also couples to the SM degrees of freedom with a Planck suppressed interaction. As in our case both the inflaton and radion are oscillating simultaneously but with different frequencies, e.g. $m_{\phi}>m_{\sigma_{\perp}}$. However inflaton being heavier will preferably decay before the radion and towards the last stage, if the radion oscillation could dominate the inflaton decay products, then, it is possible to convert isocurvature fluctuations into the adiabatic ones. Otherwise there will be a contribution from isocurvature fluctuations also. The total curvature perturbations is given by the sum of

$$
\mathcal{P}^{1 / 2} \sim f \mathcal{P}_{\sigma_{\perp}}^{1 / 2}+(1-f) \mathcal{P}_{\gamma}^{1 / 2}
$$

where $f$ is the fraction of energy density stored in the radion, it is given by

$$
f \equiv \frac{3 \Omega_{\sigma_{\perp}}}{3 \Omega_{\sigma_{\perp}}+4 \Omega_{\gamma}} .
$$

where $\Omega_{\gamma}$ is the energy density stored in the decay products of the inflaton. In our case this ratio could be small if $\Omega_{\gamma}>\Omega_{\sigma_{\perp}}$, especially since $V_{\text {inf }} \gg m_{\sigma_{\perp}}^{2} \sigma_{\perp}^{2}$ and both the fields have a similar decay rate to the SM fields. However if we imagine that not all the inflaton energy density goes into the brane degrees of freedom, suppose the inflaton couples strongly to the bulk degrees of freedom, then it is quite possible that $\Omega_{\perp} \gg \Omega_{\gamma}$. Note that even though there is a larger relativistic brane degrees of freedom, but the bulk volume is also large. Therefore it is possible to imagine that a branching ratio between brane and bulk is suppressed by the bulk volume factor.

The conclusion is that it is indeed possible to generate adiabatic density perturbations from the isocurvature fluctuations of $\sigma_{\perp}$. However a realistic scenario requires larger fundamental scale than $\mathrm{TeV}$, e.g. $M_{*} \geq 500 \mathrm{TeV}$. 


\section{B. Converting radion isocurvature fluctuations to adiabatic perturbations via fluc-} tuating inflaton coupling

There is an alternative mechanism for converting isocurvature fluctuations of $\sigma_{\perp}$ to adiabatic perturbations from fluctuating inflaton coupling. Note that in our case $\sigma_{v}$ has a natural coupling to the inflaton while $\sigma_{\perp}$ is an independent field which only couples SM degrees of freedom. Suppose the $\sigma_{v}$ has not settled down to its global minimum during inflation, which may occur if $\sigma_{v}$ has a flatter potential for both positive and negative vevs. In such a case the inflaton coupling to the SM fields will fluctuate when the inflaton decays due to the presence of the term $e^{-\alpha \sigma_{v} / M_{p}} V$ in the action. During inflation $\sigma_{v}$ has fluctuations, and if it is lighter compared to $H_{\text {inf }}$ then it also has a non-zero vev. Further the above term can give rise to fluctuating inflaton coupling or fluctuating inflaton mass. In either case the perturbations can be transferred to the SM degrees of freedom during the decay of the inflaton. This can be understood in two steps; first note that fluctuating coupling or mass gives rise to fluctuations in the decay rate of the inflaton and second, fluctuating decay rate gives rise to fluctuations in the reheat temperature of the universe. The fluctuations in $\Gamma$ can be translated into fluctuations in the energy density of a thermal bath with [47]

$$
\frac{\delta \rho_{\gamma}}{\rho_{\gamma}}=-\frac{2}{3} \frac{\delta \Gamma}{\Gamma} .
$$

The factor $2 / 3$ appears due to red-shift of the modes during the decay of the inflaton whose energy still dominates. It has already been proven that the curvature perturbation can be fed by the fluctuations in the decay rate of the inflaton $\mathcal{P}_{\zeta}^{1 / 2}=-(1 / 6) \delta \Gamma / \Gamma[47$, , 48]. Note that in this case, the tilt in the spectral index is given by Eq. (10.1).

In our case, fluctuations in the decay rate is given by

$$
\frac{\delta \Gamma}{\Gamma}=-\frac{\delta \sigma_{v}}{\sigma_{v}} \sim \frac{H_{\mathrm{inf}}}{2 \pi \sigma_{v}} .
$$

In order to match the observed COBE normalization, the vev of $\sigma_{v}$ during inflation must satisfy $\sigma_{v} \sim 10^{5} H_{\text {inf }} \approx 10^{5} M_{*}$. Since we know the initial vev of $\sigma_{v}$ at the onset of inflation has to be around $\sigma_{v}(0) \sim 10 M_{p}$, therefore one requires at least $\mathcal{N}_{e} \sim \mathcal{O}\left(10^{4}-10^{5}\right)$ number of e-foldings to bring down the vev of $\sigma_{v}$ to appreciable value in order to generate the right amplitude for the density perturbations.

In reality both the radions can play roles simultaneously; fluctuations in $\sigma_{v}$ can give rise to fluctuations in reheat temperature of the universe and late decay of $\sigma_{\perp}$ can transfer its 
isocurvature perturbations to adiabatic ones. However the dominant one will prevail and it seems that both requires large number of e-foldings of inflation, which can be obtained for the bulk inflation only, see Eq. (9.13).

\section{RADION AS A CANDIDATE FOR COLD DARK MATTER}

Since the class of models we are discussing do not have the conventional dark matter candidates such as the axion or the neutralino, we like to explore other particles as CDM. As we have noticed a successful cosmological scenario seems to point to a reheat temperature of the universe which is much smaller than the cut-off scale $M_{*}$. Therefore in order to have a stable KK dark matter 49], it is necessary to excite them non-thermally. Nevertheless we should also keep in mind that we do not understand thermalization of the inflaton decay very well and it could be that for some reason the reheat temperature is close to $T_{r} \leq M_{*}$ in which case KK modes would have no trouble getting excited and play the role of dark matter.

There however exists another interesting possibility that the radion oscillations can also act as a cold dark matter provided the radions are absolutely stable and light. According to Eq. (6.2) this happens if the radion mass is lighter than $30 \mathrm{MeV}$. Thus, if we believe in a radion potential that traps the radion in the minimum during inflation, then once $m_{\sigma_{v}} \sim H(t) \lesssim 30 \mathrm{MeV}$ or $m_{\sigma_{\perp}} \sim H(t) \lesssim 30 \mathrm{MeV}$, the radions start oscillating respectively in their minimum with an amplitude of order $H(t)$. These are coherent oscillations which give rise to an effective pressureless fluid and the density stored in their coherent oscillations can give rise to a cold dark matter. This issue will be dealt separately in near future.

\section{CONCLUSION}

In this paper we have studied some aspects of the cosmology of models with universal extra dimensions, where SM particles live on a $4+n$ dimensional brane, whereas gravity resides in $4+n+p$ dimensions (with $p$ dimensions being much larger than $n$ dimensions). These models generically have two radion fields in the effective four dimensional theory. The

two radions can be identified as (i) The volume radion, associated to the variation of the whole volume of the extra space; and (ii) the shape mode, produced by the variations in the 
hierarchy among the $n$ small and the $p$ large extra dimensions in the theory.

Our study shows the difficulties that the radions introduce in the description of early universe cosmology. In exploring this we consider two extreme forms of the radion potential. If the radion stabilization potential is rather flat, almost all scenarios of inflation suffer from a radion problem analogous to the well known moduli problem. We note that, although the radions themselves can drive inflation, the picture seems hard pressed to reconcile with the correct amplitude for the the primordial density perturbations and, typically inflation ends leaving the radions to oscillate with large amplitudes. We then focus on brane induced inflation and note that inflation occurs at relatively low scale and the inflaton energy density is typically too small (about $\mathcal{O}(\mathrm{eV})^{4}$ ) to drive a successful inflation. On the other hand, a bulk inflaton can help to settle down the volume radion to an effective minimum within a Hubble time and seems more promising from the point of view of density perturbations. Nevertheless, the effective minimum for the radion potential is usually located at large values of the radion field, which means that the bulk inflaton makes the volume of the extra dimensions to grow much beyond the expected stabilized size. If inflation is supported by both the volume radion and the inflaton, while the inflaton keeps rolling down the volume shrinks. In contrast, the shape mode gets decoupled and therefore remains frozen during the entire inflationary phase. By the end of inflation it is very likely that both the radion modes will end with large amplitudes, unless the universe passes through an extremely large number of e-foldings.

Most of the above mentioned problems get resolved if the radion mass is assumed to be large. This allows the radion to decay fast into SM particles while oscillating at late stages. With a bulk inflaton, the radion problem can disappear completely for the volume radion which is now rapidly driven to its actual minimum and trapped there by the dynamics of inflation. For this it is mandatory that the flatness of the volume modulus is lifted by some brane or bulk physics. We have explored phenomenologically this possibility.

Finally we concentrated on the radion induced isocurvature fluctuations. The observations demand that we convert this isocurvature fluctuations to the adiabatic ones. It is quite possible in our case because the radions can decay into the SM degrees of freedom. However this again would require the radion masses to be fairly large $\geq 10 \mathrm{TeV}$ at least, so they decay before BBN. Another interesting possibility which we have noticed is that the inflaton coupling to the SM matter can fluctuate, because of the couplings being governed by the 
vevs of the radions. These fluctuations induce fluctuation in the coupling and therefore in the reheat temperature of the Universe. This could be an interesting avenue to obtain the desired amplitude for the density perturbations. This particular scenario does not demand large masses for the radions, but it certainly requires fairly intermediate scale vevs for the radion mass, e.g. $\sim 10^{5} M_{*}$. This can be obtained easily if there is large e-foldings of inflation, which occurs very naturally in the bulk driven inflation scenario.

The punch line of this paper can be summarized as follows; for $\mathcal{O}(100) \mathrm{TeV}$ scale gravity the bulk driven inflation along with lifted potential for the volume radion can give rise to a successful cosmology.

\section{Acknowledgments}

A.P.L. would like to thank the Particle Theory group of the University of Maryland for the warm hospitality during the first stages of this work. The works of R. N. M. and partly of A.P.L. are supported by the National Science Foundation Grant No. PHY-0099544. A. M. is a CITA national fellow.

[1] M. Green, J. Schwarz and E. Witten, Superstring Theory, vol 1, 2, Cambridge University press, 1988; J. Polchinski, String Theory vol 1, 2, Cambridge University press, 1998.

[2] E. Witten, Nucl. Phys. B 471, 135 (1996); P. Harava and E. Witten, Nucl. Phys. B 460, 506 (1996); Nucl. Phys. B 475, 94 (1996).

[3] I. Antoniadis, Phys. Lett. B246, 377 (1990); I. Antoniadis, K. Benakli and M. Quirós, Phys. Lett. B331, 313 (1994); K. Benakli, Phys. Rev. D 60, 104002 (1999); Phys. Lett. B447, 51 (1999).

[4] N.Arkani-Hamed, S. Dimopoulos, and G. Dvali, Phys. Lett B 429, 263 (1998);Phys. Rev. D 59, 086004 (1999); I. Antoniadis, N. Arkani-Hamed, S. Dimopoulos, and G. Dvali, Phys. Lett. B 436, 257 (1998).

[5] V. A. Rubakov and M. E. Shaposhnikov, Phys. Lett. B152, 136 (1983); K. Akama, in Lecture Notes in Physics, 176, Gauge Theory and Gravitation, Proceedings of the International Symposium on Gauge Theory and Gravitation, Nara, Japan, August, 20 (1982), edited by K. 
Kikkawa, N. Nakanishi and H. Nariai, (Springer-Verlag), 267 (1983); M. Visser, Phys. Lett B159, 22 (1985); E. J. Squires, Phys. Lett B167, 286 (1986); G. W. Gibbons and D. L. Wiltshire, Nucl. Phys. B287, 717 (1987).

[6] J.C. Long and J.C. Price, hep-ph/0303057; J.C. long, et al., Nature 421, 922 (2003); C. D. Hoyle et al, Phys. Rev. Lett. , 86, 1418, (2000).

[7] G.F. Giudice, R. Rattazzi and J.D. Wells, Nucl. Phys. B544, 3 (1999); T. Han, J.D. Lykken and R.J. Zhang, Phys. Rev. D 59, 105006 (1999).

[8] See also: J.L. Hewett, Phys. Rev. Lett. 82, 4765 (1999); E.A. Mirabelli, M. Perelstein and M.E. Peskin, Phys. Rev. Lett. 82, 2236 (1999) P. Mathews, S. Raychaudhuri and K. Sridhar, Phys. Lett. B455, 115 (1999); C. Ahern, J.W. Norbury and W.J. Poyser, Phys. Rev. D 62, 116001 (2000); I. Antoniadis and K. Benakli, Int. J. Mod. Phys. A15, 4237 (2000); K. Cheung and G. Landsberg, Phys. Rev. D62, 1076003 (2000); T. Han, D. Marfatia and R.J. Zhang, Phys. Rev. D62, 125018 (2000); G. F. Giudice, A. Strumia, Nucl. Phys. B663, 377 (2003).

[9] D0 collaboration; Phys. Rev. Lett. 90 , 251802 (2003); CDF Collaboration, Phys. Rev. Lett. 89, 281801 (2002); for a review, see J. Hewett and M. Spiropulu, Ann. Rev. Nucl. Part. Sci. 52, 397 (2002) and references therein.

[10] S. Hannestad and G. Raffelt, Phys. Rev. Lett. 87, 051301 (2001); Phys. Rev. Lett. 88, 171301 (2002); Phys. Rev. D 67, 125008 (2003).

[11] R.N. Mohapatra, S. Nussinov and A. Pérez-Lorenzana, hep-ph/0308051.

[12] K.R. Dienes, E. Dudas and T. Gherghetta, Nucl. Phys. B537, 47 (1999); See also: T. Taylor and C. Veneziano, Phys. Lett. B212, 147 (1988).

[13] D. Ghilencea and G.G. Ross, Phys. Lett. B442, 165 (1998); A. Delgado and M. Quiros, Nucl. Phys. B559, 235 (1999); P.H. Frampton and A. Rasin, Phys. Lett. B460, 313 (1999); A. Pérez-Lorenzana and R.N. Mohapatra, Nucl. Phys. B559, 255 (1999).

[14] T. Appelquist, H.C. Cheng and B.A. Dobrescu, Phys. Rev. D64, 035002 (2001).

[15] B.A. Dobrescu and E. Poppitz, Phys. Rev. Lett. 87, 031801 (2001);

[16] N. Borghini, Y. Gouverneur and M.H.G. Tytgat, Phys. Rev. D65, 025017 (2002).

[17] T. Appelquist, B.A. Dobrescu, E. Ponton and H.U. Yee, Phys. Rev. Lett. 87, 181802 (2001);

[18] R.N. Mohapatra and A. Pérez-Lorenzana, Phys. Rev. D67, 075015 (2003).

[19] R.N. Mohapatra and A. Pérez-Lorenzana, Phys. Rev. D66, 035005 (2002).

[20] Y. Mimura and S. Nandi, Phys. Lett. B538, 406 (2002). 
[21] D. Lyth, Phys. Lett B 448, 191 (1999); G. Dvali, S. H. H. Tye, Phys. Lett. B450, 72 (1999); N. Kaloper and A. Linde, Phys. Rev D 59, 101303 (1999); A. Mazumdar, Phys. Lett. B 469, 55 (1999); N. Arkani-Hamed, et al., Nucl. Phys. B567, 189 (2000); J. Cline, Phys. Rev. D 61, $023513(2000)$.

[22] R.N. Mohapatra, A. Pérez-Lorenzana and C.A. de S. Pires, Phys. Rev. D 62, 105030 (1999).

[23] A. Mazumdar and A. Perez-Lorenzana, Phys. Lett. B 508, 340 (2001).

[24] A. M. Green and A. Mazumdar, Phys. Rev. D 65, 105022 (2002).

[25] T. Matsuda, Phys. Rev. D 66, 107301 (2002).

[26] M. Bastero-Gil, V. Di Clemente and S. F. King, hep-ph/0211011; hep-ph/0211012.

[27] C. Csaki, M. Graesser and J. Terning, Phys. Lett. B 456, 16 (1999).

[28] K. Benakli and S. Davidson, Phys. Rev. D 60, 025004 (1999); L. J. Hall and D. Smith. Phys. Rev. D 60, 085008 (1999).

[29] A. Mazumdar and A. Perez-Lorenzana, Phys. Rev. D 65, 107301 (2002); R. Allahverdi, K. Enqvist, A. Mazumdar and A. Perez-Lorenzana, Nucl. Phys. B 618, 277 (2001); D. J. Chung and T. Dent, Phys. Rev. D 66, 023501 (2002).

[30] C. M. Will, Theory and Experiment in Gravitational Physics, Cambridge University Press (1993).

[31] Cosmic Background Explorer homepage: http://space.gsfc.nasa.gov/astro/cobe/

[32] C.B. Netterfield et al, BOOMERANG Collaboration, Astrophys. J. 571, 604 (2002). http://www.physics.ucsb.edu/ boomerang/

[33] S. Hanany, et al, MAXIMA Collaboration, Astrophys. J. 545, L5 (2000). http://cosmology.berkeley.edu/group/cmb/

[34] C. Pryke, et al, DASI Collaboration, Astrophys. J. 568, 46 (2002). http://astro.uchicago.edu/dasi/

[35] C. L. Bennett. et.al., Astrophys. J. Suppl. 148,1 (2003). http://map.gscf.nasa.gov/

[36] H. V. Peiris et al., arXiv:astro-ph/0302225

[37] V. F. Mukhanov, H. A. Feldman and R. H. Brandenberger, Phys. Rept. 215, 203 (1992).

[38] Z. Chacko and E. Perazzi, hep-ph/0210254.

[39] N. Arkani-Hamed, L.J. Hall, D.R. Smith and N. Weiner, Phys. Rev. D 62, 105002 (2000).

[40] D. Polarski and A. A. Starobinsky, Nucl. Phys. B 385, 623 (1992). 
[41] A. R. Frey and A. Mazumdar, Phys. Rev. D 67, 046006 (2003)

[42] K. Enqvist and M. S. Sloth, Nucl. Phys. B 626, 395 (2002); D. H. Lyth and D. Wands, Phys. Lett. B 524, 5 (2002), T. Moroi and T. Takahashi, Phys. Lett. B 522, 215 (2001); [Erratumibid. B 539, 303 (2002)]; K. Enqvist, S. Kasuya and A. Mazumdar, Phys. Rev. Lett. 90, 091302 (2003); K. Enqvist, A. Jokinen, S. Kasuya and A. Mazumdar, arXiv:hep-ph/0303165.

[43] P. Jaikumar and A. Mazumdar, arXiv:hep-ph/0212265, see also R. Allahverdi, C. Bird, S. Groot Nibbelink and M. Pospelov, arXiv:hep-ph/0305010.

[44] P. Jaikumar and A. Mazumdar, arXiv:hep-ph/0309175.

[45] A. R. Liddle, A. Mazumdar and F. E. Schunck, Phys. Rev. D 58, 061301 (1998); E. J. Copeland, A. Mazumdar and N. J. Nunes, Phys. Rev. D 60, 083506 (1999).

[46] E.W. Kolb, G. Servant and T.M.P. Tait, hep-ph/0306159.

[47] G. Dvali, A. Gruzinov, and M. Zaldarriaga, astro-ph/0303591. L. Kofman, astro-ph/0303614. K. Enqvist, A. Mazumdar and M. Postma, Phys. Rev. D 67, 121303 (2003).

[48] A. Mazumdar and M. Postma, arXiv:astro-ph/0306509.

[49] G. Servant and T. M. Tait, Nucl. Phys. B 650, 391 (2003); J. L. Feng, A. Rajaraman and F. Takayama, Phys. Rev. Lett. 91, 011302 (2003). 